\title{
LENTIDÃO COGNITIVA E PSICOMOTORA EM HEMODIALISADOS CRÔNICOS
}

\author{
Wagner Martignoni de Figueiredo ${ }^{1,2}$, Ricardo de Oliveira-Souza ${ }^{1,2}$, \\ Roberto Baptista de Figueiredo ${ }^{1}$, Omar da Rosa Santos ${ }^{1}$
}

\begin{abstract}
RESUMO - Fundamentos: A encefalopatia urêmica subclínica pode levar a comprometimento ocupacional de difícil diagnóstico por requerer o emprego de medidas sensíveis. Propósito: Testar as hipóteses de que (1) pacientes em hemodiálise crônica (HDC) se saem pior do que controles normais em uma bateria de desempenho, (2) um dia extra de uremia comprometeria ainda mais o comprometimento neuropsicológico desses pacientes, e (3) a uremia dificultaria a melhora do desempenho em uma segunda sessão de testes. Método: A agilidade cognitiva e motora de 28 pacientes em HDC foi avaliada com os testes de Trilhas (A e B), Algarismos e Símbolos, e Stroop. Resultados: (1a) o desempenho cognitivo e motor se encontravam mais lento nos pacientes, (2a) um dia a mais de uremia comprometeu o desempenho na Parte B do Teste de Trilhas, e (3a) pacientes em HDC apresentaram redução da capacidade de aprender novos procedimentos. Conclusão: Pacientes em HDC podem apresentar uma "encefalopatia subclínica" cuja detecção pode requerer a aplicação de testes sensíveis. A agilidade mental e motora, e a capacidade de aprender novas rotinas estão comprometidas em, pelo menos, alguns pacientes em HDC com cognição global normal.
\end{abstract}

PALAVRAS-CHAVE: uremia, neuropsychologia, hemodiálise crônica, encefalopatia subclínica.

\section{Cognitive and psychomotor slowing in chronic hemodialysis patients}

ABSTRACT - Background: The diagnosis of "subclinical uremic encephalopathy" may need the administration of sensitive tests. Purpose: To test the hypotheses that (1) patients on chronic hemodialysis (CHD) fare worse than normal controls on a brief performance battery, (2) one extra-day of uremia further jeopardizes the neuropsychological performance of CHD patients, and (3) uremia impairs improvement on a second testing session. Method: The cognitive and motor agility of 28 patients on CHD were assessed with the Trails A and B, Digit Symbol, and Stroop tests. Results: (1a) cognitive and psychomotor performance were slowed in patients, (2a) one extra-day of uremia impaired performance further on Trail Making B, and (3a) CHD patients had a decreased ability to learn novel procedures even in the short-term. Conclusion: CHD patients may present with a "subclinical encephalopathy" whose detection may require the administration of sensible tests. Mental and motor agility, and the ability to learn new routines are impaired in at least some CHD patients with a normal global cognitive state.

KEY WORDS: uremia, neuropsychology, chronic hemodyalisis, subclinical encephalopathy.

A despeito de décadas de investigações, a busca por marcadores das manifestações da doença renal crônica tem rendido poucos frutos. A azotemia, em particular, detém correlações fracas, na melhor hipótese, com os sintomas da uremia ${ }^{1}$. Talvez isto explique, em parte, a escassez relativa de investigações sobre as manifestações neurológicas da uremia e condições associadas, como a encefalopatia hipertensiva. Não obstante, o aumento da prevalência da doença renal crônica, principalmente na população idosa, alimenta a necessidade de conhecermos melhor suas manifestações, sobretudo nos estágios iniciais, quando alterações cognitivas e comportamentais su- tis freqüentemente passam despercebidas. As manifestações neurológicas de pacientes em hemodiálise crônica (HDC), em particular, impõem desafios diagnósticos e terapêuticos únicos, devido à heterogeneidade de condições que comumente a ela se associam e que podem resultar de, pelo menos, quatro fatores distintos: à "uremia" e alterações metabólicas a ela diretamente associadas, como hipercalcemia; a efeitos iatrogênicos da $\mathrm{HDC}^{3}$; a doenças cardiovasculares coincidentes ${ }^{4}$; e a doenças neurodegenerativas concomitantes, como doença de Alzheimer ${ }^{5}$. A identificação da(s) causa(s) que atua(m) em cada caso requer experiência e sensibilidade clínica, assim como

\footnotetext{
${ }^{1}$ Escola de Medicina e Cirurgia, Universidade do Estado do Rio de Janeiro, Rio de Janeiro RJ, Brasil; ${ }^{2}$ Programa de Pós-Graduação
} em Neurologia e Neurociências da Universidade Federal Fluminense, Niterói RJ, Brasil.

Recebido 19 Março 2007. Aceito 20 Junho 2007.

Dr. Omar da Rosa Santos - Serviço de Clínica Médica C - Hospital Universitário Gaffré e Guinle - Rua Maris e Barros 775 - $20270-004$ Rio de Janeiro RJ - Brasil. E-mail: ricaredo@walla.com 
o emprego judicioso dos métodos complementares a serviço do diagnóstico diferencial. Neste sentido, exames auxiliares de uso ambulatorial e de baixo custo serão sempre bem-vindos, desde que sua utilidade clínica seja previamente estabelecida.

A síndrome que resulta dos efeitos da uremia sobre o sistema nervoso central é denominada "encefalopatia urêmica"2. Plenamente instalada, o diagnóstico não constitui problema, principalmente quando a encefalopatia se desenvolve em horas ou dias. Nos casos não tratados, o coma é precedido por confusão e períodos alternantes de sonolência e agitação acompanhados de movimentos involuntários anormais caracterizados por tremor, asterixis, mioclonias multifocais e crises convulsivas generalizadas 6 . $\mathrm{Na}$ uremia crônica, essas alterações são menos óbvias e, como ocorreu nos nossos pacientes, sua detecção requer a aplicação de testes mais sensíveis do que a mera constatação de normalidade da orientação em consultas de rotina.

A expressão "encefalopatia subclínica" ou "subliminar" congrega as alterações cognitivas que ocorrem nas encefalopatias metabólicas que ficam aquém do limiar diagnóstico de confusão mental aguda e demência. Originalmente aplicado à encefalopatia hepática ${ }^{7}$, o conceito foi estendido à uremia em investigação sobre o desempenho neuropsicológico de urêmicos crônicos ${ }^{8}$. Como em outras encefalopatias subclínicas ${ }^{9,10}$, a característica essencial da encefalopatia urêmica subclínica (EUS) é a lentidão cognitiva e psicomotora, mais acentuadas em pacientes em HDC e, em menor grau, naqueles submetidos a transplante renal. Esses resultados concordam que a capacidade de sustentar a atenção e a velocidade do processamento mental são as funções cognitivas mais sensíveis à uremia crônica não tratada ${ }^{11}$.

A presente investigação teve por objetivo verificar as hipóteses de que testes de desempenho de aplicação fácil e rotineira são capazes de detectar (1) o efeito da uremia sobre o desempenho cognitivo, (2) o efeito de incrementos discretos de uremia, mesmo que resultam do acúmulo de produtos do catabolismo em 24 horas sem diálise, e (3) o efeito da uremia sobre a capacidade de aquisição de novas habilidades, fenômeno conhecido em neuropsicologia clínica como "efeito de prática"12.

\section{MÉTODO}

Os dados foram coletados na Unidade de Hemodiálise do Setor de Nefrologia do Hospital Universitário Gaffrée e Guinle de maio a setembro de 1996 em 28 pacientes (11 mulheres e 17 homens) em HDC há, pelo menos, 8 meses (média=34 \pm 24 meses) devido a doença renal crônica ter- minal cujas causas foram estabelecidas em 21 casos: lupus eritematoso sistêmico (2), mieloma múltiplo (1), rins policísticos (2), glomerulonefrite crônica (2), glomerulonefrite crescêntica (2), hipertensão arterial sistêmica (12). Todos se encontravam clinicamente estáveis e sem intercorrências pelo 3 meses que antecederam o estudo. O programa dialítico consistia de 3 sessões semanais (terças, quintas e sábados) de banhos com bicarbonato de sódio e água tratada com deionização de 4 horas de duração em máquinas tipo tanque equipadas com capilares de cuprofane com $1,5 \mathrm{~m}^{2}$ de superfície. A eficácia dialítica foi calculada pelo índice de remoção de uréia (IRU) ao término de cada sessão, pela seguinte fórmula (na época, considerava-se "eficaz" a diálise que resultasse em IRU $>40 \%$ ):

$$
\operatorname{IRU}(\%)=1-\left(\frac{\text { uréia pós-diálise }}{\text { uréia pré-diálise }}\right) \times 100
$$

Foram excluídos pacientes com: a) vírus da hepatite B ou $C$, ou alteração nas provas funcionais hepáticas, b) intercorrências dialíticas graves (hemotransfusão, infecções ou ganho de peso levando a congestão pulmonar) nos 3 meses precedentes, c) IRU $<40 \%$ na diálise imediatamente precedente à aplicação dos testes, e d) depressão, ansiedade, confusão mental aguda e demência, segundo os critérios da quarta edição do Manual Diagnóstico e Estatístico dos Distúrbios Mentais ${ }^{13}$.

Vinte e nove indivíduos saudáveis serviram voluntariamente de controles normais $(\mathrm{CN})$, a maior parte auxiliares de enfermagem do serviço de diálise que aceitaram participar como voluntários não remunerados. Todos os participantes forneceram consentimento informado por escrito antes do ingresso no estudo.

Testes neuropsicológicos - Os testes foram aplicados por um dos autores (RBF) em ambiente silencioso e adequadamente iluminado. O Mini-Exame do Estado Mental (MEEM) foi usado no rastreamento de demência e estado confusional agudo ${ }^{14}$. Os testes de desempenho empregados foram as partes A (tTA) e B (tTB) do teste de Trilhas, as duas partes do teste de Stroop (denominação de cores e interferência palavra-cor), e o subteste de Substituição de Símbolos por Algarismos (tSA) da bateria de Wechsler ${ }^{13}$. Uma modificação do tSA em que o participante era solicitado a simplesmente copiar os símbolos na mesma seqüência do tSA, o Teste de Cópia de Símbolos (tCS), foi usada para medir apenas o componente visuomotor do tSA. Os testes de desempenho ("performance", do original em inglês) diferem dos demais testes neuropsicológicos pela inclusão do tempo de execução no cálculo das pontuações finais ${ }^{15}$ e são, por isso, cronometrados. Os resultados do desempenho são expressos como "rendimento comportamental", i.e., a razão entre precisão (número de acertos) e tempo de execução (medido em segundos). Devido a falhas na execução do projeto, os resultados do teste de Stroop foram descartados.

Desenho experimental - Cada paciente foi submetido a três testes de desempenho antes da diálise, em duas sessões de testes. Na primeira (Np1), realizada quinta-feira, 
o paciente se encontrava a 44 horas da diálise precedente (realizada na terça-feira). Na segunda (Np2), terça-feira, o paciente se encontrava a 68 horas da diálise precedente (realizada no sábado). Por ocasião de Np2, portanto, os pacientes se encontravam, aproximadamente, 24 horas "mais urêmicos". Os controles foram submetidos ao mesmo protocolo para (1) contrastar o efeito da uremia e da HDC sobre o desempenho, e (2) estimar o "efeito de prática" de cada teste, ou seja, o grau de aprendizado promovido pela repetição dos testes após curto intervalo. O efeito de prática foi operacionalmente definido como o ganho de desempenho na segunda execução de cada teste pelos controles, correspondendo à simples subtração dos pontos em Np1 de Np2 (ou seja: Np2-Np1). Para ajustar o efeito de prática, a mediana dos efeitos de prática nos controles (tTA $=-3$ seg, tTB $=-19$ seg, tSA $=-0,01$, e tCS $=-0,10$ ) foi subtraída das pontuações dos pacientes, e o novo resultado (Np2') empregado nas análises subseqüentes. Em tese, portanto, variações estatisticamente significativas no desempenho dos pacientes de Np1 a Np2' não poderiam ser atribuídas à prática, sendo, portanto, melhor explicadas por efeitos inerentes à HDC.

Análise estatística ${ }^{16}$ - Os resultados numéricos estão descritos como médias e desvios-padrão. Associações entre variáveis categóricas foram estudadas pelo teste do quiquadrado $\left(\chi^{2}\right)$. A significância das diferenças entre as médias dos grupos (controles $x$ pacientes) foi examinada por análises de variância (ANOVA), usando o teste de Scheffé nas comparações post hoc entre pares de médias. A significância das comparações entre os resultados das avaliações
Np1 ("menos urêmico") e Np2 ("mais urêmico") foi estudada por análises de variância para medidas repetidas, uma vez que, neste caso, cada indivíduo foi seu próprio controle. Utilizamos o coeficiente de correlação de Pearson ( $r$ ) para verificar associações entre variáveis neuropsicológicas e tempo de HDC. Finalmente, procedemos à regressão logística para determinar a importância relativa das variáveis dependentes na afiliação dos participantes a cada grupo. Os cálculos foram efetuados no Statistical Package for the Social Sciences para Windows, v. 13 (Apache Software Foundation, 2004). Adotamos o limiar de significância convencional $(\alpha=0,05)$, bicaudado, exceto nas correlações, por causa do número de comparações múltiplas, em que optamos pelo limiar mais restrito de Bonferroni $(\alpha=0,004)$.

\section{RESULTADOS}

Os resultados numéricos estão descritos na Tabela 1. Os grupos diferiram em idade, escolaridade, e composição de sexo. As discrepâncias de idade e escolaridade foram estatisticamente corrigidas por análises de covariância (ANCOVA) em todas as comparações entre os grupos.

Efeito da uremia sobre o desempenho cognitivo no contexto da HDC - Não houve diferença significativa entre controles e pacientes quanto ao estado cognitivo global, conforme avaliado pelo MEEM $\left(F_{1,53}=0,64, p>0,43\right)$. Os IRU das duas sessões de diálise estiveram acima do limiar de eficácia dialítica (varia-

Tabela 1. Dados demográficos e resultados dos testes.

\begin{tabular}{|c|c|c|c|}
\hline & & Controles & HDC \\
\hline $\mathrm{N}$ (mulheres/homens) & & $29(27 / 2)^{a}$ & $28(11 / 17)^{\mathrm{b}}$ \\
\hline Idade (anos) & & $35 \pm 9$ & $41 \pm 11^{c}$ \\
\hline Escolaridade (anos) & & $9,5 \pm 2,8$ & $7,1 \pm 2,8^{c}$ \\
\hline \multicolumn{4}{|l|}{ Eficácia da hemodiálise } \\
\hline \multirow[t]{2}{*}{ IRU (1-100) } & Np1 & - & $59,7 \pm 7,5$ \\
\hline & $\mathrm{Np2}$ & - & $59,3 \pm 9,1^{d}$ \\
\hline \multicolumn{4}{|l|}{ Estado cognitivo global } \\
\hline Mini-exame do estado mental (0-30) & & $27,7 \pm 1,8^{e}$ & $26,9 \pm 2,7$ \\
\hline \multicolumn{4}{|l|}{ Testes de desempenho } \\
\hline \multirow[t]{3}{*}{ Teste de trilhas A (tTA) } & Np1 & $44,1 \pm 18,7$ & $67,9 \pm 35,6$ \\
\hline & $\mathrm{Np2}$ & $39,1 \pm 12,9^{d}$ & $65,6 \pm 37,3$ \\
\hline & Np2' & - & $68,6 \pm 37,2$ \\
\hline \multirow[t]{3}{*}{ Teste de trilhas B (tTB) } & Np1 & $115,3 \pm 50,1$ & $140,9 \pm 57,7$ \\
\hline & $\mathrm{Np} 2$ & $101,5 \pm 57,6^{d}$ & $159,6 \pm 66,5$ \\
\hline & $\mathrm{Np2}$ & - & $178,6 \pm 66,5$ \\
\hline \multirow[t]{3}{*}{ Teste de substituição de algarismos por símbolos (tSA) } & Np1 & $2,11 \pm 0,57$ & $3,27 \pm 1,52$ \\
\hline & $\mathrm{Np2}$ & $2,01 \pm 1,52^{f}$ & $2,90 \pm 1,56$ \\
\hline & $\mathrm{Np2}$ & - & $2,91 \pm 1,56$ \\
\hline \multirow[t]{3}{*}{ Teste de cópia de símbolos (tCS) } & Np1 & $1,01 \pm 0,25$ & $1,092 \pm 1,068$ \\
\hline & Np2 & $0,89 \pm 0,22^{f}$ & $1,44 \pm 0,55$ \\
\hline & $\mathrm{Np2}$ & - & $1,54 \pm 0,55$ \\
\hline
\end{tabular}

${ }^{a} \chi^{2}$ para amostra única: $p<0,01 ;{ }^{b} \chi^{2}$ para amostra única: não significante; ${ }^{\mathrm{c} A N C O V A}$ (controles $x$ pacientes), Schefée: $p<0,01$; ${ }^{\mathrm{d}} \mathrm{ANCOVA}$ para medidas repetidas (Np2-Np1): não significante; e ANCOVA (controles x pacientes), Schefée: não significante; ${ }^{\dagger} A N C O V A$ para medidas repetidas (Np2-Np1): $\mathrm{p}<0,05$; Np1, primeira testagem neuropsicológica (quinta-feira); Np2, segunda testagem neuropsicológica (terça-feira); Np2', segunda testagem neuropsicológica descontados os efeitos de prática. 
Tabela 2. Proporção de indivíduos com melhor desempenho Np2'.

\begin{tabular}{lcc}
\hline & Controles & HDC \\
\hline tTA & $16 / 28(57 \%)$ & $15 / 27(56 \%)$ \\
tTB & $29 / 29(100 \%)^{*}$ & $5 / 27(19 \%)^{* \#}$ \\
tSA & $19 / 29(66 \%)$ & $17 / 28(61 \%)$ \\
tSC & $24 / 27(89 \%)^{*}$ & $14 / 28(50 \%)^{\#}$ \\
\hline
\end{tabular}

${ }^{*}$ Teste binominal: $p<0,01 ;{ }^{*} C N x H D C\left(\chi^{2}\right): p<0,001$.

ção de IRU 1=44-71\%; variação de IRU 2=45-76\%), e tampouco variou significativamente entre as sessões de $\operatorname{HDC}\left(t_{1,27}=0,27, p>0,78\right)$, indicando que as sessões de diálise tiveram eficácia semelhante. As correlações entre tempo de HDC e desempenho neurocognitivo não foram significativas e não serão relatadas.

Análise complementar foi conduzida para averiguar se as diferenças entre os grupos permaneceriam depois de "descontado" o efeito de prática embutido na segunda testagem. Comparando novamente os resultados em $\mathrm{Np2}$ entre os grupos, agora covariando os resultados em $\mathrm{Np} 1$, verificamos que as diferenças se mantiveram para tTA $(p<0,0001)$ e tTB $(p<0,0001)$, para o tSA $(p<0,0001)$ e para o tCS $(p<0,0001)$, indicando que o efeito de prática nos pacientes não foi suficiente para normalizar seu desempenho.

Efeito da uremia sobre o desempenho cognitivo repetido - Quando o desempenho nos dois momentos de teste foram comparados em cada grupo, pacientes e controles diferiram em um aspecto essencial: enquanto nos controles o desempenho médio melhorou de Np1 para Np2 em todos os testes, refletindo os ganhos esperados, nos pacientes este efeito não foi significativo, tanto nas comparações Np2Np1 como Np2'-Np1. Para ter uma idéia mais clara da freqüência do efeito de prática em cada teste, classificamos o desempenho em Np2 como "melhor" (1), "igual" (0) ou "pior" (-1) do que Np1. Como mostrado na Tabela 2, a prática melhorou o desempenho da maior parte dos controles em todos os testes. O mesmo foi observado nos pacientes, mas, neste caso, a melhora não foi significativa. Além disso, mais controles apresentaram efeito de prática na parte $B$ do teste de trilhas e no TCS.

Procedemos, então, à regressão logística binomial entrando grupo (pacientes $x$ controles) como variável dependente para determinar os padrões de associação das seguintes covariáveis: sexo, idade, escolaridade, MEEM e melhora do desempenho na segunda aplicação dos testes de desempenho. Embora, em conjunto, essas variáveis se correlacionassem com o grupo ao qual o indivíduo pertencia $\left(R^{2}=0,64\right.$, $p<0,001)$, a variância da associação foi determinada pelo sexo masculino $(O R=0,06, p<0,04)$, menor escolaridade $(O R=0,62, p<0,03)$ e piora na repetição da parte $B$ do teste de Trilhas ( $O R=13,6, p<0,03$ ).

\section{DISCUSSÃO}

O estudo demonstrou que pacientes em HDC por insuficiência renal terminal, clinicamente estáveis e com cognição global normal (i.e., sem confusão mental ou demência), apresentam lentidão cognitiva e psicomotora. Além disso, um dia a mais de uremia comprometeu o desempenho no tTB. Finalmente, os pacientes tiveram dificuldade de aprender com a prática, uma vez que não apresentaram os ganhos de desempenho, evidentes nos controles, quando testados pela segunda vez. Esta diferença se deveu tanto à intensidade do efeito de prática, quanto à proporção de indivíduos que o manifestaram.

A importância da EUS em pacientes em HDC é realçada pela freqüência com que passa despercebida. Em estudo recente, alterações cognitivas acometeram $87 \%$ de 338 pacientes em HDC, mas foram documentadas pela equipe clínica em apenas $3 \%{ }^{17}$. Esses resultados e os nossos concordam que a normalidade da cognição global, aferida pelo MEEM, não afasta a possibilidade de disfunção cerebral que, em muitos casos, se manifesta por lentidão cognitiva e psicomotora, inaparentes à observação casual, mas detectáveis por testes suficientemente sensíveis. Nossa impressão de que a lentidão precede o declínio cognitivo global foi indiretamente confirmada em estudo comparando pacientes com doença renal crônica e em $\mathrm{HDC}^{18}$. Neste trabalho, a doença renal crônica desses pacientes foi estratificada, com base na mediana do clearance (TFG) estimado, em levea-moderada (TFG $\geq 25,5 \mathrm{ml} / \mathrm{min} / 1,73 \mathrm{~m}^{2}$ ) e adiantada (TFG $<25,5 \mathrm{ml} / \mathrm{min} / 1,73 \mathrm{~m}^{2}$ ). Os autores observaram declínio cognitivo global (avaliado pelo MEEM) na doença renal crônica adiantada e nos pacientes em HDC, mas não na doença renal crônica leve-a-moderada. Em contraste, o desempenho (avaliado pela parte B do teste de Trilhas) foi mais lento nos três subgrupos.

$\mathrm{Na}$ época em que nossa investigação foi concluída, os critérios de adequação dialítica eram menos restritivos e poderiam justificar a EUS. As alterações de desempenho podem ter-se devido ao considerado "ineficaz" para os atuais padrões de eficácia dialítica. Esta idéia é apoiada por estudos mais recentes, que se utilizaram de controles mais rigorosos de eficácia do tratamento. Por exemplo, Umans e Pliskin aplicaram seis testes de desempenho (três deles os mesmos que usamos no presente trabalho) a uma pequena 
amostra de pacientes em HDC idosos e de alta escolaridade ${ }^{19}$. O ingresso na pesquisa exigia que todos estivessem clinicamente estáveis e "bem dialisados", ou seja, com hematócrito acima de $30 \%$ e depuração fracional de uréia superior a 1 durante os 6 meses precedentes. A comparação dos resultados desses pacientes com os dos controles confirmou que, quando a eficácia dialítica é rigorosamente controlada, não há diferença estatística entre pacientes e controles em nenhum dos seis testes. Os autores concluíram que a lentidão cognitiva relatada em pacientes em HDC são passíveis de correção por diálises que preencham os critérios correntes de eficácia.

No cenário da redução progressiva da TFG, a fisiopatologia da EUS pode refletir (a) apenas o grau mínimo de encefalopatia ou (b) uma síndrome independente, com fisiopatologia própria. A primeira possibilidade é apoiada pela progressão ao coma da uremia não tratada ${ }^{20}$. A segunda, pela existência de pacientes urêmicos igualmente graves, mas que não entram em coma, manifestando, em seu lugar, graus extremos de lentidão psicomotora e cognitiva (estupor catatônico).

A prática modifica o comportamento como "memórias implícitas". Em contraste com a memória explícita ou declarativa (que o indivíduo adquire em uma única exposição, acessa voluntariamente, e expressa conscientemente por meios verbais ou simbólicos), a memória implícita ou de procedimentos, medeia hábitos e habilidades (andar de bicicleta, tocar piano, dirigir). Sua aquisição e aprimoramento depende de treinamento e prática. Estes domínios da memória podem ser comprometidos por lesões cerebrais em locais diferentes ${ }^{21}$. Nossos resultados sugerem que a capacidade de aprender pela prática pode estar comprometida em pacientes em HDC. Se esta hipótese for confirmada, a aplicação de testes de desempenho poderia ajudar na indicação de procedimentos que dependem da participação ativa do próprio paciente. Esta idéia foi sugerida há alguns anos em hemodialisados ambulatoriais ${ }^{22}$. Usando a bateria de Wechsler, os autores verificaram que, apesar do QI normal, os pacientes estavam comprometidos em três subtestes de desempenho, um dos quais o tSA, o mesmo que utilizamos em nossos casos. Complementaram o estudo transformando o subteste de Desenhos com Cubos ("block design") em tarefa de memória implícita com 5 ensaios repetidos. $O$ achado mais instigante foi $o$ de que a deterioração do efeito de prática nos Desenhos com Cubos prenunciou declínio cognitivo.

O tTB piorou em mais de $80 \%$ dos pacientes, indicando que os mecanismos neurais subjacentes à alternância cognitiva (no caso, entre letras e números) devem estar debilitados na HDC. Resultado seme-
Ihante foi obtido em um dos raros estudos com testes repetidos aplicados a pacientes em $\mathrm{HDC}^{23}$. Dos testes que empregamos, o tTB é o que mais recruta as redes cerebrais executivas ${ }^{24}$, particularmente organizadas pelos córtices frontal (dorsolateral e anteromedial) e parietal. A maior sensibilidade do tTB na HDC indica que o desempenho executivo se encontra precocemente comprometido na EUS. O impacto da EUS na vida dos pacientes urêmicos segue pouco conhecido e constitui objeto de investigações em curso.

Agradecimentos - A Jorge Baçal e a José Ricardo Pinheiro (Biblioteca do Instituto Osvaldo Cruz) pela atenção e presteza com que atendem às nossas solicitações bibliográficas.

\section{REFERÊNCIAS}

1. Fraser CL, Arieff AJ. Nervous system complications in uremia. Ann Intern Med 1988;109:143-153.

2. Burn DJ, Bates D. Neurology and the kidney. J Neurol Neurosurg Psychiatry 1998;65:810-821

3. Ratner DP, Adams KM, Levin NW, Rourke BP. Effects of hemodialysis on the cognitive and sensory-motor functioning of the adult chronic hemodialysis patient. J Behav Med 1983;6:291-311.

4. Lass P, Buscombe JR, Harber M, Davenport A, Hilson AJ. Cognitive impairment in patients with renal failure is associated with multiple-infarct dementia. Clin Nucl Med 1999;24:561-565.

5. Seliger S. Risk factors for incident stroke among patients with end-stage renal disease. J Am Soc Nephrol 2003;14:2623-2631.

6. Lockwood AH. Neurologic complications of renal disease. Neurol Clin 1989;7:617-627.

7. Rikkers L, Jenko P, Rudman D. Subclinical hepatic encephalopathy: detection, prevalence, and relationship to nitrogen metabolism. Gastroenterology 1978;75:462-469.

8. Figueiredo RB. Encefalopatia urêmica subclínica em pacientes hemodialisados: comparação entre testes neurocognitivos. Tese. Niterói, 2000.

9. Gray C, Cantagallo A, Della Salla S, Basaglia N. Bradykinesia and bradyphrenia revisited: patterns of subclinical deficit in motor speed and cognitive functioning in head-injured patients with good recovery. Brain Inj 1998;12:429-441.

10. Haggerty JJ, Garbutt JC, Evans DL, et al. Subclinical hypothyroidism: a review of neuropsychiatric aspects. Int J Psychiatry Med 1990;20:193-208.

11. Hart RP, Pederson JA, Czerwinski AW, Adams RL. Chronic renal failure, dialysis, and neuropsychological function. J Clin Neuropsychol 1983;5:301-312.

12. Lezak M. Neuropsychological assessment. 3.Ed. New York: Oxford University Press, 1995.

13. American Psychiatric Association. Diagnostic and statistic manual of mental disorders, 4.Ed. (DSM-IV). Washington: American Psychiatric Association, 1994.

14. Brucki SMD, Nitrini R, Caramelli P, Bertolucci PHF, Okamoto IH. Sugestões para o uso do mini-exame do estado mental no Brasil. Arq Neuropsiquiatr 2003;61:777-781.

15. Oliveira-Souza R, Moll J, Eslinger PJ. Neuropsychological assessment. In Rizzo M, Eslinger PJ (Eds). Principles and practice of behavioral neurology and neuropsychology. New York: Elsevier, 2004:47-64.

16. Welkowitz J, Ewen RB, Cohen J. Introductory statistics for the behavioral sciences. Philadelphia: Harcourt Brace Jovanovich, 1986.

17. Murray AM, Tupper DE, Knopman DS, et al. Cognitive impairment in hemodialysis patients is common. Neurology 2006;67:216-223.

18. Kurella M, Chertow GM, Luan J, Yaffe K. Cognitive impairment in chronic kidney disease. J Am Geriatr Soc 2004;52:1863-1869.

19. Umans JG, Pliskin NH. Attention and mental processing speed in hemodialysis patients. Am J Kidney Dis 1998;32:749-751.

20. Plum F. Coma and related global disturbances of the human conscious state. In Peters A (Ed). Cerebral cortex, volume 9: Normal and altered states of function. New York Plenum Press, 1991:359-425.

21. Budson AE, Price BH. Memory dysfunction. New Eng J Med 2005;352: 692-699.

22. English A, Savage RD, Britton PG, Ward MK, Kerr DN. Intellectual impairment in chronic renal failure. Br Med J 1978;1:888-890.

24. Moll J, Oliveira-Souza R, Moll FT, Bramati IE, Andreiuolo PA. The cerebral correlates of set-shifting. An fMRI study of the trail making test. Arq Neuropsiquiatr 2002;60:900-905. 